\title{
Safety, efficacy, and onset of a novel botulinum toxin type A (Nabota) for the treatment of glabellar frown lines: a single-arm, prospective, phase 4 clinical study
}

\author{
Sinyoung Song, \\ Yeon Hoon Lee, \\ Joon Pio Hong, \\ Tae Suk Oh \\ Department of Plastic and \\ Reconstructive Surgery, Asan Medical \\ Center, University of Ulsan College of \\ Medicine, Seoul, Korea
}

\begin{abstract}
Background: Safety, efficacy, and time to onset of effect of botulinum toxin type A is of importance to persons who seek improvement in glabellar frown lines, but this has not been well studied. The aim of this study was to determine the safety, efficacy, and onset of action of a newly developed botulinum toxin type A (Nabota) for the treatment of glabellar frown lines.

Methods: This was a single-arm, open-label, and phase 4 clinical study. Forty-two subjects with glabellar lines were treated with five times of intramuscular injection of $0.1 \mathrm{~mL}(4 \mathrm{U} / 0.1 \mathrm{~mL})$ for a total of $20 \mathrm{U}$ of Nabota. Efficacy and safety were assessed at 2, 3, 4, 5, and 14 days. Efficacy was assessed by the investigator and it was defined as a 1-point change on a 4-point scale.

Results: Improvement in glabellar frown lines at maximum frown was observed in $85.4 \%$ of subjects 2 days after administration. Improvement in glabellar lines at rest was observed in $51.2 \%$ of subjects 2 days after administration, and the proportion of subjects showing improvement increased with time. No severe adverse events were recorded.

Conclusion: Onset of action was observed in the majority of subjects by 2 days after administration of Nabota. In addition, Nabota was found to be safe and effective for the treatment of glabellar frown lines.
\end{abstract}

Keywords: Botulinum toxins, type A / Glabellar frown line / Safety / Efficacy / Onset

\section{INTRODUCTION}

Glabellar frown lines are considered to be cosmetically undesirable, and they can be aggravated by aging, anger, or worry. Botulinum toxin A, produced from Clostridium botulinum, relaxes the muscles by inhibiting the neurotransmitter acetylcholine

\footnotetext{
Correspondence: Tae Suk Oh

Department of Plastic and Reconstructive Surgery, Asan Medical Center,

University of Ulsan College of Medicine, 88 Olympic-ro 43-gil, Songpa-gu,

Seoul 05505, Korea

E-mail: tasuko@amc.seoul.kr

*This study was sponsored by Daewoong Pharmaceutical.

Received May 8, 2018 / Revised June 29, 2018 / Accepted July 5, 2018
}

and is widely used as a local muscle relaxant [1-3]. Botulinum toxin A is also used for aesthetic concerns [4-9], such as glabellar frown lines, crow's feet, and smile lines, as well as forehead transverse lines. Several formulations of botulinum toxin type A are available for cosmetic use, including onabotulinumtoxi$\mathrm{nA}$, abobotulinumtoxin $\mathrm{A}$, and incobotulinumtoxin $\mathrm{A}$, among which onabotulinumtoxinA has been studied the most. A new botulinum product, Nabota (DWP450-004; Daewoong Pharmaceutical, Seoul, Korea), which is a Botulinum toxin type A derived from the same strain of $C$. botulinum as onabotulinumtoxinA has been developed. In addition to efficacy, the rapidity of effect may affect patient satisfaction in facial rejuvenation. 
Indeed, the onset of effect is an important factor that can influence a subject's level of satisfaction with their cosmetic treatments. The aim of the present study was to precisely determine for Nabota the time-point during the first 2 weeks after treatment at which the onset of effect occurs. One limitation of previous studies of the time of onset of action of Botulinum toxins was judgment based on self-evaluation by subjects $[10,11]$. Therefore, the present study investigated the time to onset of treatment effect of Nabota in the treatment of glabellar frown lines as determined by a physician using a 4-point scale, as well as examining its efficacy and safety.

\section{METHODS}

\section{Subjects and eligibility criteria}

This study was a single-arm, open-label, single-institute, domestic, and phase 4 clinical study. Subjects who desired improvement of their glabellar frown lines and had provided written informed consent were screened with respect to the inclusion/exclusion criteria based on their medical history (Fig. 1). The target number of study subjects was calculated to be 40 patients, in consideration of the significance level of 5\%; a $95 \%$ confidence interval with a range of $20 \%$ was permitted, and the above ratio of improvements in glabellar frown lines. Finally, considering the ratio of $10 \%$ of halfway dropouts, the target number of study subjects was 45 patients. Therefore, the number of study subjects needed for this clinical trial was planned to be a total of 45 patients.

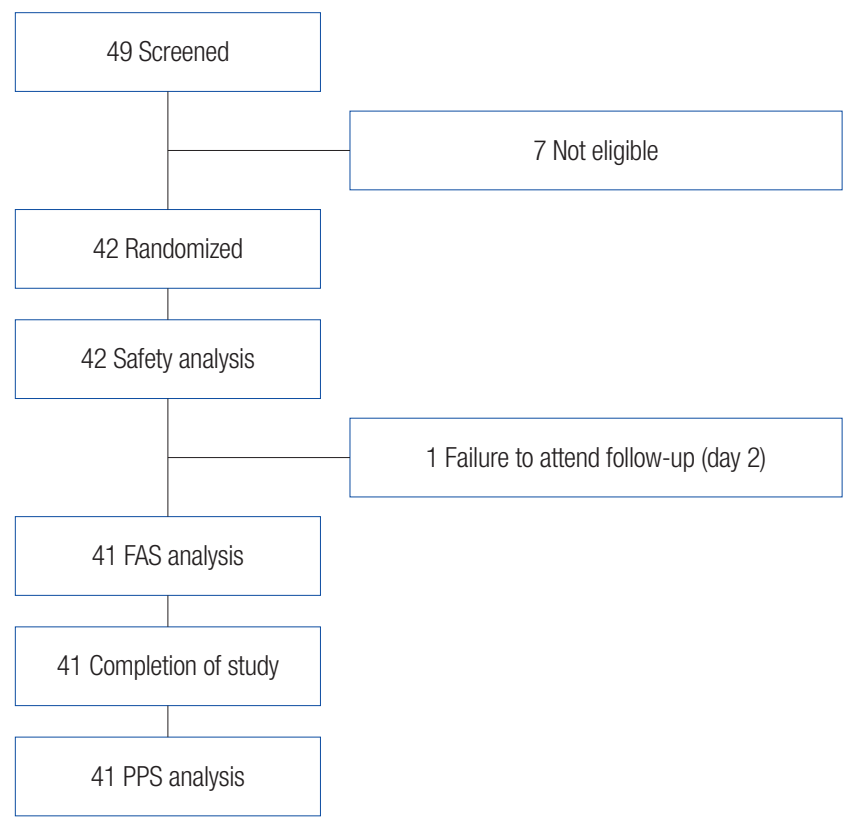

Fig. 1. Subject disposition. FAS, full analysis set; PPS, per protocol set.

$$
\begin{gathered}
N=\frac{4 p(1-p) \times Z_{1-\alpha / 2}^{2}}{d^{2}} \\
\approx 40
\end{gathered}
$$

where, $\mathrm{N}$ is the number of patients; $p, 0.88 ; \alpha, 0.05$ (95\% confidence level); $d, 0.2$.

Written consent was obtained for the publication of participants' photos in this article. The study was approved by the Institutional Review Board of the Asan Medical Center (IRB No. 2014-0128). The study was registered at ClinicalTrials.gov (NCT02568150).

Subjects were eligible to participate in the study if their age was between 20 and 65 years, with an investigator-assessed glabellar frown line score of 2 or 3 at maximum frown on a 4-point facial wrinkle scale of 0 (none), 1 (mild), 2 (moderate), and 3 (severe) (Table 1). Subjects who had undergone other procedures that could affect the assessment of wrinkles on or around the forehead in the 6 months prior to screening were excluded. Subjects with skin abnormalities, such as infection at the planned injection site, skin disease, or scar; those with a previous history of facial palsy or ptosis; those with allergies to the contents of the injection, those with contraindications to Botulinum toxins treatment (including pregnancy and breast feeding); those with severe concomitant diseases; and those whose circumstances did not allow regular participation in the study were also excluded.

\section{Procedure}

A volume of $2.5 \mathrm{~mL}$ of $0.9 \%$ sodium chloride was used to dilute $100 \mathrm{U}$ of Nabota to allow administration of $4 \mathrm{U}$ in $0.1 \mathrm{~mL}$. Each subject was injected intramuscularly with $0.1 \mathrm{~mL}$ of Nabota into two sites near the corrugator muscle (total of four locations) and into one site at the procerus muscle (total of five locations) (Fig. 2). A $0.5 \mathrm{~mL}$ of the investigational product was injected so that each subject received a total of $20 \mathrm{U}$. After administration, observation was carried out for 30 minutes to detect adverse events.

\section{Assessment of efficacy}

The primary efficacy and the secondary efficacy variables were the proportion of subjects with improvement in glabellar frown

Table 1. Facial wrinkle scale at maximum frown

\begin{tabular}{lll}
\hline Score & Severity & \multicolumn{1}{c}{ Explanation } \\
\hline 3 & Severe & $\begin{array}{r}\text { Wrinkles are clearly visible. The depth of wrinkles } \\
\text { cannot be assessed from the surface. } \\
2\end{array}$ \\
$\begin{array}{lll}\text { Wrinkles are clearly visible. The depth of wrinkles } \\
\text { can be assessed from the surface. }\end{array}$ \\
0 & Mild & Wrinkles are visible. \\
\hline
\end{tabular}




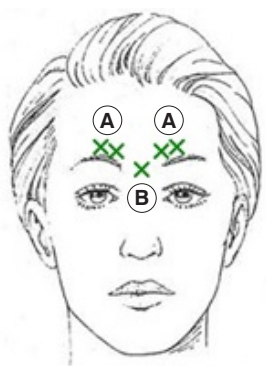

\begin{tabular}{|l|c|c|}
\hline & Injection unit & Injection volume \\
\hline $\begin{array}{c}\text { A: Corrugator muscle (injected at 2 } \\
\text { points [total of 4 points] bilaterally) }\end{array}$ & $16 \mathrm{U}$ (8 U/corrugator muscle) & $0.4 \mathrm{~mL}(0.1 \mathrm{~mL} /$ injection site) \\
\hline B: Procerus muscle (injected at 1 point) & $4 \mathrm{U}$ & $0.1 \mathrm{~mL}$ \\
\hline Total dose & $20 \mathrm{U}$ & $0.5 \mathrm{~mL}$ \\
\hline
\end{tabular}

Fig. 2. Injection sites.

Table 2. Facial wrinkle scale at rest

\begin{tabular}{lll}
\hline Score & Severity & \multicolumn{1}{c}{ Explanation } \\
\hline 3 & Severe & Wrinkles are clearly visible. \\
2 & Moderate & Wrinkles are visible. \\
1 & Mild & Some wrinkles are visible. \\
0 & None & Wrinkles are not visible. \\
\hline
\end{tabular}

lines as assessed by a single expert investigator using photography when the subject wrinkles his or her glabellar region at maximum frown and at rest at 2, 3, 4, 5, and 14 days after Nabota administration. The photography was taken from five angles (front, both three-quarters oblique views, both lateral views) at rest and at maximum frown. The photo shows the patients' vertex to the sternal head of clavicle. During taking photography, the patients were educated to locate their earlobes perpendicularly and centrally, the horizontal line was aligned based on the level of labial commissure, eyebrow. In threequarters oblique view, the nasal tip was located in line with the contour of cheeks. Efficacy was based on the investigator's assessment of glabellar line severity at rest and at maximum frown using a 4-point facial wrinkle scale: 0 (none), 1 (mild), 2 (moderate), and 3 (severe). Improvement was defined as a decrease of 1 point on the 4-point scale compared with baseline (Tables 1,2). The exploratory efficacy assessment variables were the number of subjects with an improvement in glabellar frown lines when the subject wrinkled his/her glabella at maximum frown and at rest at 2, 3, 4, 5, and 14 days after administration of Nabota.

\section{Safety}

Adverse events and adverse drug reactions, both serious and unexpected, were assessed. Causal relationship to the investigational product was evaluated by the physician in charge using six steps that are based on the assessment criteria.
Table 3. Characteristics of study participants

\begin{tabular}{lc}
\hline Characteristic & Subject $(\mathrm{n}=41)$ \\
\hline Age (yr), mean \pm SD (range) & $45.51 \pm 10.26(26-60)$ \\
Sex & $5(12.2)$ \\
Male & $36(87.8)$ \\
Female & \\
Glabellar lines at maximum frown & 0 \\
0 (None) & 0 \\
1 (Mild) & $28(68.29)$ \\
2 (Moderate) & $13(31.71)$ \\
3 (Severe) & \\
Glabellar lines at rest & $3(7.32)$ \\
0 (None) & $16(39.02)$ \\
1 (Mild) & $15(36.59)$ \\
2 (Moderate) & $7(17.07)$ \\
3 (Severe) & \\
\hline
\end{tabular}

Values are presented as number (\%) unless otherwise indicated. $\mathrm{SD}$, standard deviation.

\section{Statistical analysis}

Data were divided into three forms for analysis: safety analysis, full analysis set (FAS), and per protocol set. In all analyses, including the assessment of subject characteristics, efficacy assessment, and safety assessment, continuous variables were expressed as a descriptive quantity (observed subject number, mean \pm standard deviation, and median). Frequency and percentages were also calculated for categorical variables such sex. All statistical analyses were performed using SAS (Enterprise Guide 4.3; SAS Institute Inc., Cary, NC, USA). A two-tailed test was performed, a was set as $5 \%$.

\section{RESULTS}

In total, 42 subjects were enrolled in the current study. The assessment result of glabellar frown lines on frowning during visit 3 (2 days after administration) was dropped out in one case, so it was excluded from the FAS analysis.

\section{Baseline characteristics}

Baseline data of the 41 subjects included in the FAS are presented in Table 3. The assessment of glabellar frown lines at maximum frown during screening revealed that 28 subjects (68.3\%) had a score of 2 and 13 subjects (37.7\%) had a score of 3. Assessment of glabellar frown lines at rest at the time of screening revealed that three subjects (7.3\%) had a score of 0, $16(39.0 \%)$ had a score of $1,15(36.6 \%)$ had a score of 2 , and seven (17.1\%) had a score of 3 . The age of subjects that had a score of 0 was 20-29 years, and they were all female.

\section{Primary efficacy assessment variable}

The proportion of subjects with improvement of glabellar 
frown lines at maximum frown as assessed by the investigator was $85.4 \%$ (35 subjects) at 2 days after administration of the investigational product; $85.4 \%$ (35 subjects) at 3 days after administration; $90.2 \%$ (37 subjects) at 4 days after administration; 95.1\% (39 subjects) at 5 days after administration; and $97.6 \%$ (40 subjects) at 14 days after administration (Fig. 3). Thus, an increasing proportion of subjects experienced improvement with time. $2.4 \%$ (one subject) of subjects experienced no improvement after administration of the investigational product even after 14 days.

\section{Secondary efficacy assessment variable}

The proportion of subjects who showed an improvement in glabellar frown lines at rest as assessed by the investigator was $51.2 \%$ (21 subjects) at 2 days after administration of the product; $58.5 \%$ (24 subjects) at 3 days after administration; $63.4 \%$ (26 subjects) at 4 days after administration; $78.1 \%$ (32 subjects)

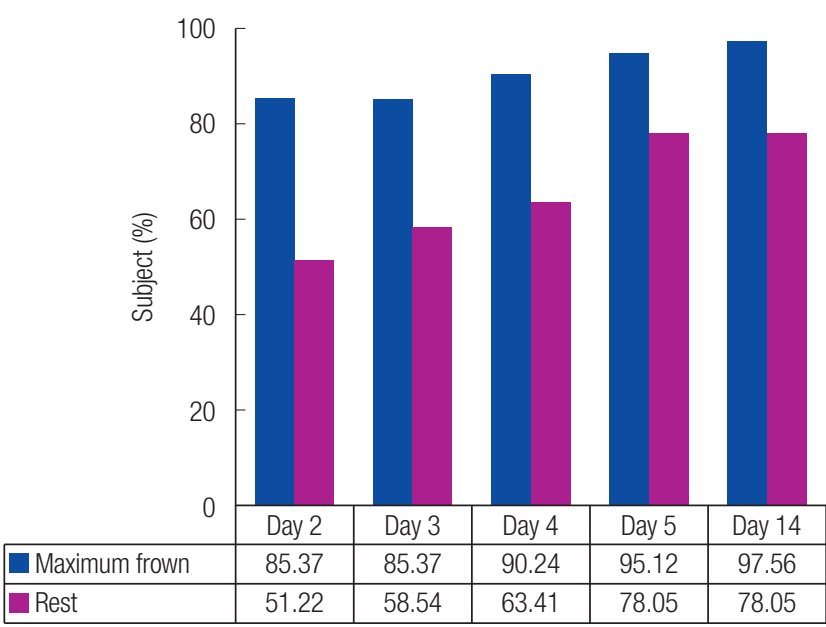

Fig. 3. Glabellar line improvement proportion at maximum frown and rest based on the investigator's evaluation. The Y-axis shows the percentage of subjects who responded with a 1-point decrease from baseline as evaluated by the investigator.

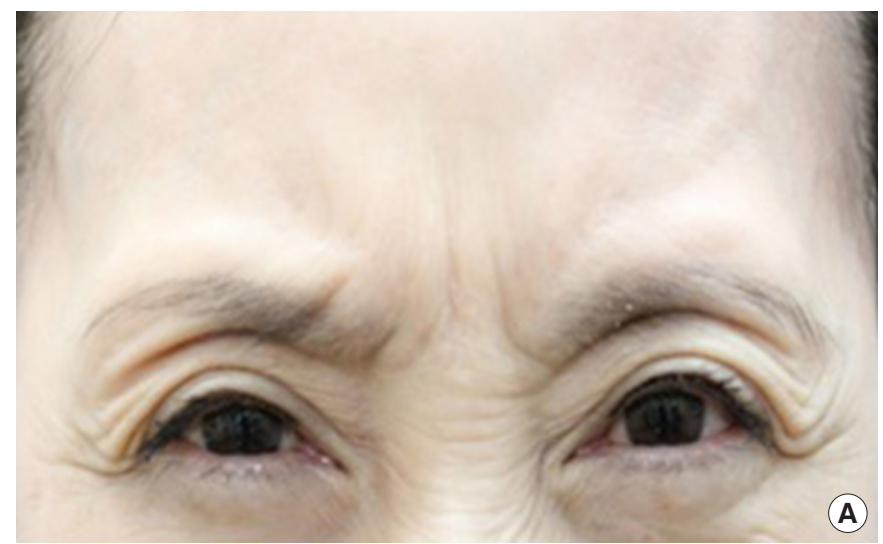

at 5 days after administration; and $78.1 \%$ (32 subjects) at 14 days after administration (Fig. 3). Thus, the proportion of subjects who experienced an improvement tended to increase with time. Nine subjects (21.9\%) experienced no improvement after administration of the investigational product even after 14 days.

\section{Explorative efficacy assessment variable}

The proportion of subjects with an initial improvement of glabellar frown lines after the administration of the investigational product at maximum frown, as assessed by the investigator, was $85.4 \%$ (35 subjects) at 2 days, $0 \%$ at 3 days, $4.9 \%$ (two subjects) at 4 days, $4.9 \%$ (two subjects) at 5 days and $2.4 \%$ (one subjects) at 14 days (Table 4 ). This result showed most subjects had an initial improvement 2 days after administration of the investigational product. Figs. 4 and 5 show two representative subjects of different ages with improvement of glabellar lines at maximum frown 2 days after Nabota injection.

The proportion of subjects with an initial improvement of glabellar frown lines at rest after administration was 51.2\% (21 subjects) at 2 days, 7.3\% (three subjects) at 3 days, $4.9 \%$ (two subjects) at 4 days, $14.6 \%$ (six subjects) at 5 days and $0 \%$ at 14 days (Table 5). These results show that approximately $78 \%$ of subjects showed an improvement between 2 and 5 days after administration of the investigational product.

Table 4. Proportion of subjects with improvement of glabellar lines at maximum frown based on investigator's assessment

\begin{tabular}{ccc}
\hline Day after administration & No. (\%) & $95 \% \mathrm{Cl}$ \\
\hline 2 & $35(85.37)$ & $70.83-94.43$ \\
3 & 0 & 0 \\
4 & $2(4.88)$ & $0.6-16.53$ \\
5 & $2(4.88)$ & $0.6-16.53$ \\
14 & $1(2.44)$ & $0.06-12.86$ \\
\hline
\end{tabular}

$\mathrm{Cl}$, confidence interval.

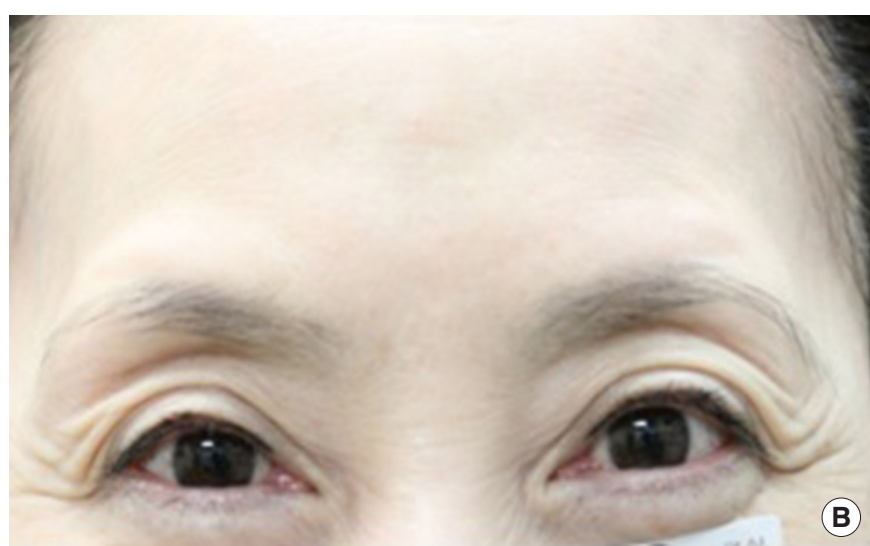

Fig. 4. Noted improvement of glabellar lines at maximum frown 2 days after injection. (A) Day 0, 2 points. (B) Day 2, 0 points. 

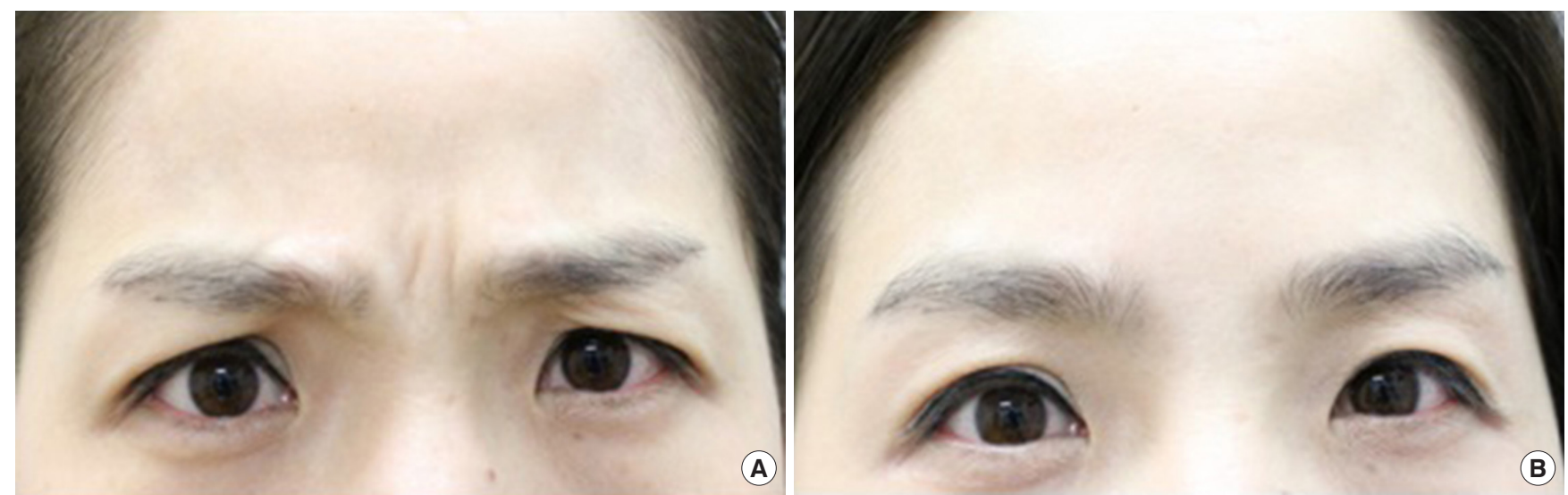

Fig. 5. Noted improvement of glabellar lines at maximum frown 2 days after injection. (A) Day 0, 2 points. (B) Day 2, 0 points.

Table 5. Proportion of subjects with improvement of glabellar lines at rest based on investigator's assessment

\begin{tabular}{ccr}
\hline Day after administration & No. (\%) & \multicolumn{1}{c}{$95 \% \mathrm{Cl}$} \\
\hline 2 & $21(51.22)$ & $35.13-67.12$ \\
3 & $3(7.32)$ & $1.54-19.92$ \\
4 & $2(4.88)$ & $0.6-16.53$ \\
5 & $6(14.63)$ & $5.57-29.17$ \\
14 & 0 & 0 \\
\hline
\end{tabular}

Cl, confidence interval.

\section{Safety}

Of the 42 subjects, four experienced mild adverse events (9.5\%) such as oropharyngeal pain, rhinitis allergic, folliculitis, and pruritus that were not related to the investigational product, spontaneously resolved. There were no severe adverse events that significantly disturb daily activities of the study subjects and have harmful effect on the health, such as headache, lumbar radiculopathy, tremor. And there were no reports of adverse events related to the investigational product within 2 weeks of Nabota administration. Thus, the safety of the administration of Nabota for glabellar frown lines was confirmed.

\section{DISCUSSION}

Nabota has been shown to be more effective than Botox in improving glabellar frown lines. A randomized, double-blind, phase 3 study comparing Nabota with onabotulinumtoxinA in the treatment of moderate to severe glabellar lines showed better responder rates at 4 weeks after Nabota treatment (93.9\% vs. 88.6\%) for lines at maximum frown, while the incidence of adverse events were similar between the two treatment groups (20\% vs. 18.1\%) [9]. The study thus demonstrated similar efficacy and safety between Nabota and onabotulinumtoxinA. The time to onset of action of Botulinum toxins in the treatment of facial rhytides, among other factors, is important to persons who seek facial rejuvenation; they may have a preference for a product with a rapid onset and a fast-acting product, as it would likely contribute to their satisfaction with treatment. However, there is limited information on the time to onset of treatment effect. The purpose of the current study was to examine the onset of improvement of glabellar frown lines and safety of a novel Botulinum toxin type A (Nabota) for adults who desired improvement of moderate-to-severe glabellar frown lines. This study evaluated the improvement of frown lines by using a facial wrinkle scale (4-point scale). In the study by Beer et al. [10], the effect of onabotulinumtoxinA on glabellar lines was documented by almost half of subjects by day 1 , with $87 \%$ of subjects showing improvement as assessed by a physician on day 2 . A subanalysis of three studies of abobotulinumtoxinA revealed that $19.7 \%$ of participants documented an effect on glabellar lines on day 1 with median onset at day 3 [11]. After incobotulinumtoxinA injection, the majority of subjects experienced improvement in glabellar lines at maximum frown 2 to 4 days after treatment, with $95.2 \%$ and $85.0 \%$ of subjects experiencing $\geq 1$-point and $\geq 2$-point improvement on a 5 -point scale as assessed by an independent rater using photos [12]. Because the reported proportion of improvement is not constant on the first day after administration, we decided to investigate onset time by the second day after injection. The effect of Nabota on glabellar frown lines at maximum frowning was observed at 2 days after administration in $85.4 \%$ of subjects, and the proportion of subjects with improvement increased with time. The median time of primary efficacy onset was 2.3 days. Similarly, the effect of Nabota on improvement of glabellar frown lines at rest was initially observed 2 days after the administration of the investigational product in $51.2 \%$ of subjects, and the proportion of subjects with improvement increased with time. The median time of secondary efficacy onset was 2.8 days.

Three formulations of Botulinum toxin type A have been ap- 
proved for use in the United States and Europe for the treatment of glabellar rhytides; namely, onabotulinumtoxinA, abobotulinumtoxin $\mathrm{A}$, and incobotulinumtoxin $\mathrm{A}$, for which a few studies have been conducted to examine the time to onset of effect. In one study of onabotulinumtoxinA injection (20 U) for glabellar frown lines by Beer et al. [10], the time to onset was determined by physicians, and subjects were asked to answer yes or no on whether they had noticed any effect on the appearance of the frown lines after the injection. Physician assessment showed that $87 \%, 91 \%$, and $100 \%$ of subjects had an effect 2,3 , and 4 days after the injection, respectively, while the corresponding percentages for subject-reported rates were $77 \%$, $93 \%$, and $98 \%$, as recorded in a 14-day diary [10]. Notably, almost half of the subjects reported onset by day 1 . These results are consistent with the results of our study, demonstrating that the majority of subjects experience an effect by day 2 post-injection. A study of onabotulinumtoxinA treatment for hyperfunctional facial lines, including glabellar frown lines, reported that all subjects noticed an effect within the first 24-72 hours, as assessed by both subjects and physicians using a 0 to 3 rating scale [13]. Our finding was interesting because improvement at rest took longer to achieve than improvement at maximum frown. This finding has many possible implications. Perhaps a higher dose is needed to achieve better resting improvement, or perhaps it will be necessary to educate patients about the delayed effects of Nabota on wrinkles present at rest. Nevertheless, it is apparent that Nabota starts working as early as day 1 after administration, with the majority of patients showing a positive response by day 2 .

Improvement in glabellar lines was also observed with other formulations of botulinum toxin type A. Based on the results of phase 3 trials of abobotulinumtoxinA (50-80 U) for the treatment of glabellar frown lines [11], an integrated analysis of these trials revealed that $19.7 \%$ of subjects noticed an effect after 1 day, with the median time to onset of 3 days, and more than $50 \%$ of subjects experienced improvements in glabellar frown lines 2-4 days after administration. However, these findings were based on subject assessment only. Two of these trials were fixed-dose studies. At 2, 3, and 7 days posttreatment, the cumulative percentages of respondents were 55\%, 74\%, and $90 \%$, respectively, in the first study, and $35.2 \%, 52.4 \%$, and $80 \%$, respectively, in the second study. A study of 23 subjects who received incobotulinumtoxinA on the glabella showed that $95.2 \%$ and $85.0 \%$ of subjects had $\geq 1$-point and $\geq 2$-point improvement on a 5-point scale as assessed by an independent rater, respectively, in glabellar frown lines at maximum frown 2-4 days after treatment. It was found that $84 \%$ of the maximum effect occurred in this time interval. The proportion of subjects who had at least a 1-point improvement at maximum frown was $100 \%$ in the two subsequent visits around days 8 and 14 [12]. It is difficult to compare the rapidity of effect of different formulations of botulinum toxin type A for the treatment of glabellar lines because of the different study designs. Rappl et al. [14] attempted to address this in a randomized, double-blind study by comparing onabotulinumtoxinA (21 U), abobotulinumtoxinA (63 U), and incobotulinumtoxinA (21 U) in 180 subjects (60 in each group). Assessment by the injecting physician showed that the median times to onset in female subjects were 3.02, 5.29, and 5.32 days for incobotulinumtoxin A, onabotulinumtoxin A, and abobotulinumtoxinA, respectively, while the corresponding times for males were 3.36, 5.89, and 5.93 days, indicating that incobotulinumtoxinA had the fastest onset times in both sexes between the toxin formulations.

With regard to safety in the present study, four of the 42 subjects (9.52\%) experienced treatment-emergent adverse events, all of which were mild and were not related to the investigational product. None of the subjects dropped out of the study because of an adverse event. Our safety data confirmed that Nabota was well tolerated by subjects and are consistent with those in other studies [11-13].

The present study has notable limitations. First, it was not a comparative, randomized, controlled study. Second, it did not assess the duration of effect, and time to return to pretreatment wrinkle severity (relapse rate) was not examined. Finally, although the assessment of efficacy was performed using photography by a single expert investigator, the evaluation criteria of wrinkles' severity is not clear enough to remove bias. Further study of these factors would achieve more quantitative results. Nabota was confirmed to be safe and effective with a fast onset time. Nabota represents a valuable addition to existing Botulinum toxins products.

\section{CONFLICT OF INTEREST}

Dr. Joon Pio Hong and Dr. Tae Suk Oh serve as consultants and lecturers for Daewoong Pharmaceutical. This study was sponsored by Daewoong Pharmaceutical. The sponsor was involved in the collection, analysis, and interpretation of data; writing the report; and the decision to submit the report for publication. No other potential conflict of interest relevant to this article was reported.

\section{PATIENT CONSENT}

The patients provided written informed consent for the publication and the use of their images. 


\section{REFERENCES}

1. Tsui JK. Botulinum toxin as a therapeutic agent. Pharmacol Ther 1996;72:13-24.

2. Pearce LB, First ER, MacCallum RD, Gupta A. Pharmacologic characterization of botulinum toxin for basic science and medicine. Toxicon 1997;35:1373-412.

3. Kim CS, Jang WS, Son IP, Nam SH, Kim YI, Park KY, et al. Electrophysiological study for comparing the effect of biological activity between type A botulinum toxins in rat gastrocnemius muscle. Hum Exp Toxicol 2013;32:914-20.

4. Harii K, Kawashima M. A double-blind, randomized, placebocontrolled, two-dose comparative study of botulinum toxin type A for treating glabellar lines in Japanese subjects. Aesthetic Plast Surg 2008;32:724-30.

5. Honeck P, Weiss C, Sterry W, Rzany B; Gladys study group. Reproducibility of a four-point clinical severity score for glabellar frown lines. Br J Dermatol 2003;149:306-10.

6. Carruthers JD, Lowe NJ, Menter MA, Gibson J, Eadie N; Botox Glabellar Lines II Study Group. Double-blind, placebo-controlled study of the safety and efficacy of botulinum toxin type A for patients with glabellar lines. Plast Reconstr Surg 2003; 112:1089-98.

7. Carruthers JA, Lowe NJ, Menter MA, Gibson J, Nordquist M, Mordaunt J, et al. A multicenter, double-blind, randomized, placebo-controlled study of the efficacy and safety of botulinum toxin type A in the treatment of glabellar lines. J Am Acad Dermatol 2002;46:840-9.

8. Won CH, Lee HM, Lee WS, Kang H, Kim BJ, Kim WS, et al. Efficacy and safety of a novel botulinum toxin type A product for the treatment of moderate to severe glabellar lines: a randomized, double-blind, active-controlled multicenter study. Dermatol Surg 2013;39(1 Pt 2):171-8.

9. Won CH, Kim HK, Kim BJ, Kang H, Hong JP, Lee SY, et al. Comparative trial of a novel botulinum neurotoxin type A versus onabotulinumtoxin $\mathrm{A}$ in the treatment of glabellar lines: a multicenter, randomized, double-blind, active-controlled study. Int J Dermatol 2015;54:227-34.

10. Beer KR, Boyd C, Patel RK, Bowen B, James SP, Brin MF. Rapid onset of response and patient-reported outcomes after onabotulinumtoxinA treatment of moderate-to-severe glabellar lines. J Drugs Dermatol 2011;10:39-44.

11. Schlessinger J, Monheit G, Kane MA, Mendelsohn N. Time to onset of response of abobotulinumtoxina in the treatment of glabellar lines: a subset analysis of phase 3 clinical trials of a new botulinum toxin type A. Dermatol Surg 2011;37:1434-42.

12. Prager W, Bee EK, Havermann I, Zschocke I. Onset, longevity, and patient satisfaction with incobotulinumtoxinA for the treatment of glabellar frown lines: a single-arm, prospective clinical study. Clin Interv Aging 2013;8:449-56.

13. Blitzer A, Binder WJ, Aviv JE, Keen MS, Brin MF. The management of hyperfunctional facial lines with botulinum toxin: a collaborative study of 210 injection sites in 162 patients. Arch Otolaryngol Head Neck Surg 1997;123:389-92.

14. Rappl T, Parvizi D, Friedl H, Wiedner M, May S, Kranzelbinder B, et al. Onset and duration of effect of incobotulinumtoxi$\mathrm{nA}$, onabotulinumtoxin $\mathrm{A}$, and abobotulinumtoxin $\mathrm{A}$ in the treatment of glabellar frown lines: a randomized, double-blind study. Clin Cosmet Investig Dermatol 2013;6:211-9. 